Journal for ImmunoTherapy of Cancer

\title{
Do the benefits of being a smoker hint at the existence of PD-1/PD-L1 sensitizers for patients on single- agent immunotherapy?
}

\author{
Gintaras Zaleskis, ${ }^{1}$ Vita Pasukoniene, ${ }^{1}$ Dainius Characiejus, ${ }^{2}$ Vincas Urbonas ${ }^{3}$
}

To cite: Zaleskis G, Pasukoniene V, Characiejus D, et al. Do the benefits of being a smoker hint at the existence of PD-1/PD-L1 sensitizers for patients on single-agent immunotherapy? Journal for ImmunoTherapy of Cancer 2021;9:e003191. doi:10.1136/ jitc-2021-003191

Accepted 09 August 2021
Check for updates

(c) Author(s) (or their employer(s)) 2021. Re-use permitted under CC BY-NC. No commercial re-use. See rights and permissions. Published by BMJ.

${ }^{1}$ Laboratory of Immunology, Nacionalinis vezio institutas, Vilnius, Lithuania

${ }^{2}$ Medical Faculty, Vilnius University, Vilnius, Lithuania ${ }^{3}$ Laboratory of Clinical Oncology, Nacionalinis vezio institutas, Vilnius, Lithuania

Correspondence to Dr Gintaras Zaleskis; gintaras.zaleskis@nvi.lt

\begin{abstract}
Multiple studies demonstrate significantly better therapeutics outcomes in smokers as compared with never smokers when single-agent immunotherapy is applied. Non-smoker patients usually need a combination of chemoimmunotherapy to achieve comparable or slightly better therapeutic results. This effect is thought to be due to tobacco product-induced upregulation of PD-L1/PD-1 expression and tumor mutational burden score. Genomic transformation, however, cannot entirely explain the upregulation of PD-L1/PL-1 expression in cells following short-term exposure to cytotoxic compounds. Cytotoxic drugs, crude tobacco products, benzo(a)pyrene, nicotine, and multiple other toxic compounds were shown to exhibit rapid PD-L1/PD-1 upregulation. A significant immunomodulatory effect of nicotine via acetylcholine receptors is well documented. However, nicotine activity rapidly subsides when the drug is withdrawn. We hypothesize that smoking cessation might mitigate the benefits of monoimmunotherapy for some patients. Further studies of the nicotinic acetylcholine receptor stimulus of immunocytes are needed and might lead to characterization and clinical implementation of new immunotherapy sensitizer products.
\end{abstract}

Shall we insist on smoking cessation for terminal non-small cell lung cancer (NSCLC) patients with a very poor general condition? Best supportive care rather than compassionate immunotherapy is the standard of care for these patients and most of them will die within 1-4 months of diagnosis. Nevertheless, a recent study ${ }^{1}$ showed that patients with NSCLC with a poor general condition can sometimes derive long-term benefits from single-agent immunotherapy. However, there were two parameters that projected the ineffectiveness of the author's therapeutic setting: (a) brain metastases and (b) never/lower level of smoking history. These findings are in agreement with another surprising observation $^{2}$ showing that smokers can benefit from single-agent immunotherapy, whereas nonsmokers need additional preconditioning chemotherapy.
A correlation between smoking history and a higher PD-L1 tumor proportion score is well documented and was assumed to be due to a higher mutational frequency. ${ }^{3}$ Yet, there is a resistance to acknowledge that a fraction of patients with NSCLC might actually benefit from the continuation of smoking once a diagnosis is established. This counterintuitive argument is supported by recent findings elucidating the fine mechanisms of PD-1/PD-L1 induction. The upregulation mechanism of the PD-1/PD-L1 axis appears to function through an unspecific response to almost any toxin exposure in a mode of danger signal adaptation. The majority of other anticancer agents, nicotine and even benzo(a)pyrene, ${ }^{4}$ share this unusual PD-1/ PD-L1 upregulating capacity. Could it not be that tobacco products are as efficient as cytotoxic drugs in maintaining a high score of PD-1/PD-L1 in the tumor microenvironment? It is a fact well known that any agent which is cytotoxic can cause cancer and can be used for cancer therapy precisely because it is cytotoxic. In addition, tobacco products and benzo(a)pyrene, in particular, were shown to induce enrichment of $\mathrm{CD} 4^{+}, \mathrm{CD}^{+}$, and PD- $1^{+}$lymphocytes in the lungs of mice. ${ }^{4}$ The discontinuation of antigenic or toxic stimulation might lead to the cessation of earlier induced upregulation of PD-1/PD-L1. The stimulus mediated by smoking resembles metronomic therapy where low doses of anticancer drugs are given on a continuous, regular schedule usually over a long period of time. Metronomic chemotherapy causes less severe side effects than conventional chemotherapy. Very poor condition smokers after an NSCLC diagnosis might simply need the continuation of 'metronomic smoking' to keep their status quo with an increased PD-1/ PD-1L expression. Accidentally, the same toxic metabolite, acrolein, is accumulating 
in cells and tissues of patients effectively treated with cyclophosphamide or long-term smokers. Is metronomic cyclophosphamide really less harmful than 'metronomic smoking'?

It is impossible to ignore the evidence of certain health benefits of smoking cessation for patients with cancer. However, the absolute majority of these data were obtained on patients who were not exposed to a single-agent immunotherapy regimen. Amazing as it may seem, single-agent immunotherapy needs precisely one of the two preconditioning stimuli: (a) chemotherapy or (b) chronic exposure to tobacco products (figure 1A,Bsomewhat similar concept is advocated by Mo et $a l^{2}$ ). Both stimuli-chemotherapy and smoking-are carcinogenic. The exploration of each of these stimuli for a patient with NSCLC contains its pros and cons. Platinum-based

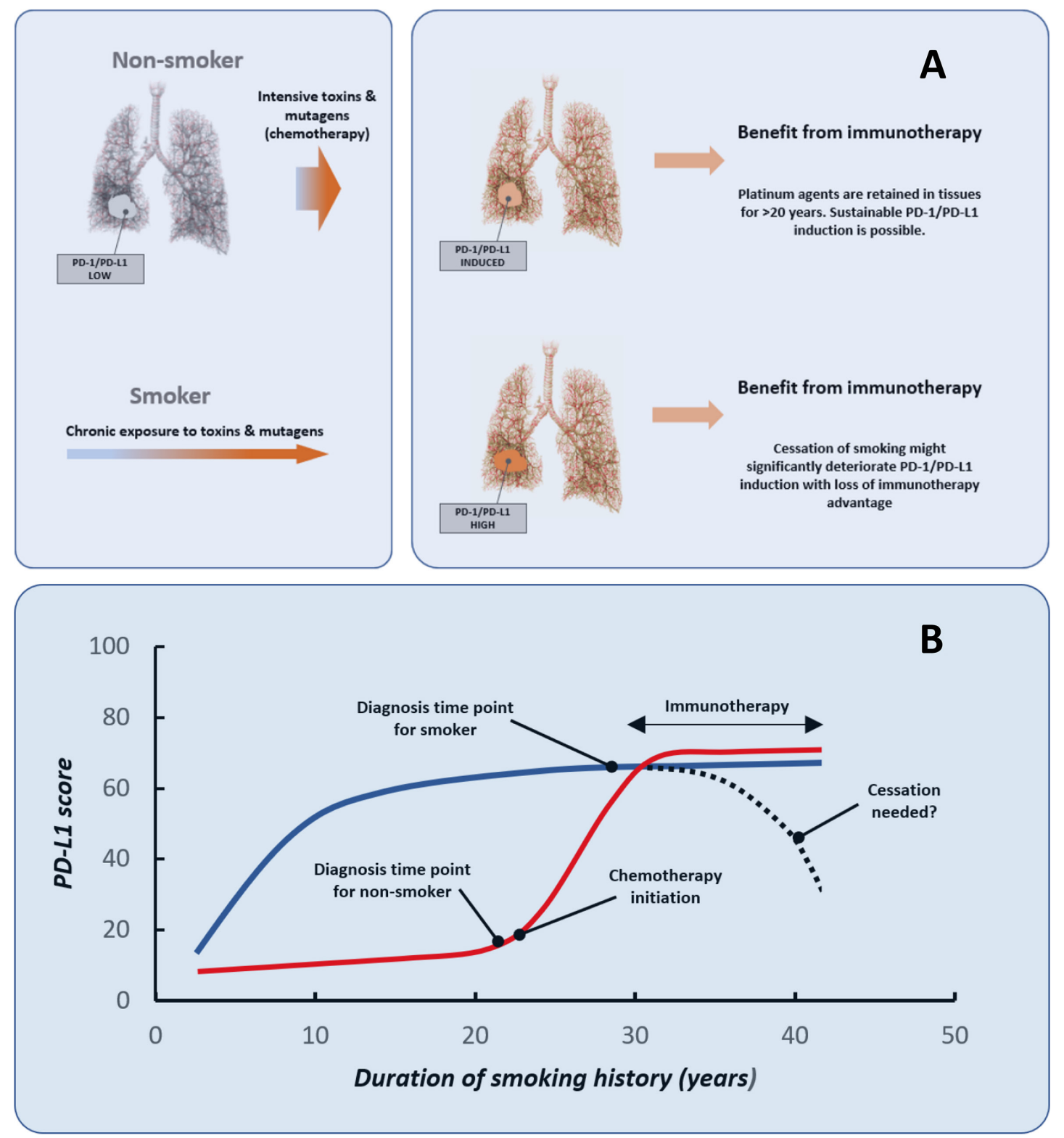

Figure 1 (A) Two different scenarios demonstrating the involvement of tissue 'danger signals' in the regulation of immunotherapy response. Long-term tobacco exposure or chemotherapy is bringing patients to a similar preconditioned state which is eventually rendering a therapeutic response to immunotherapy. The toxic and mutagenic agents from various exposures (eg, nicotine, cisplatin, carboplatin, pemetrexed, benzo(a)pyrene, acrolein, and possibly many other tobacco products) are inducing 'danger signals' in tumor or immune cells. This results in the upregulation of PD-1/PD-L1. Smokers then appear to benefit from monoimmunotherapy. Conversely, non-smokers need additional 'toxin and mutagen' stimulus elicited by cytotoxic drugs. This generates a false impression that non-smokers are representing a different group of patients who benefit most from 'combination chemoimmunotherapy'. (B) The time frame of the same two scenarios. Smokers (blue line) on initiation of immunotherapy will benefit if their PD-L1/PD-1 expression is already high and the stimulus of upregulation (smoking) is not discontinued. Conversely, chemotherapy-induced upregulation in non-smokers (red line) might be significantly more stable than tobacco product-induced upregulation. This chemotherapy-induced long-term effect might be due to platinum agent retention in tissues for $>20$ years. 
chemotherapy elicits severe side effects. However, platinum-based chemotherapy is unique since platinum compounds can be retained in the patient's tissues for many years. ${ }^{5}$ Therefore, tissue fixed platinum can also maintain a high PD-1L/PD-1 score for a longer period of time. Residual body indwelling platinum cytotoxicity could be demonstrated for at least 3 years after treatment. ${ }^{5}$ Conversely, tobacco product exposure needs to be applied continuously to elicit its specific effect on cells.

Smoking-induced tissue damage is rather reversible after cessation. It means that smoking cessation might reduce the intensity of danger signals needed for PD-1/ PD-L1 expression. Healthcare providers frequently overestimate the risks associated with nicotine, mistakenly perceiving nicotine as the main carcinogen in cigarettes and a leading cause of smoking-related diseases. However, carcinogen-induced lung cancer development needs at least 5-20 years of the latency period. So, smoking cessation for a very poor condition patient with an estimated life expectance of 1-4 months does not make much sense, especially if a dramatic life extension in a fraction of these patients is a small but still realistic opportunity. ${ }^{1}$ Smoking cessation for patients selected for monoimmunotherapy is hard to rationalize on the basis of the currently available biomarker evidence.

Most patients quit or attempt to quit shortly after a cancer diagnosis, but still up to $50 \%$ of them continue to smoke. Withdrawal symptoms might provoke sleep disorders, depression, suicidal thoughts, weight gain, metabolic insulin resistance, and other well-reported complications. Tobacco products can sometimes elicit an unusual effect on several autoimmune diseases. For instance, there is overwhelming epidemiological evidence indicating that smoking protects against ulcerative colitis, an inflammatory disease that increases the risk of colorectal cancer. ${ }^{6}$ Former smokers who resume smoking after a diagnosis of ulcerative colitis often experience clinical improvement. However, these patients cannot be cured by smoking renewal. The medical community is unwilling to admit that controversies related to the tobacco product immunomodulating activity are a reality, not a hypothesis. The majority of publications are presenting indistinct data illustrating the therapeutic benefits of immunotherapy in heavy smokers regardless of tumor type. A characteristic example can be seen in some publications ${ }^{7}$ revealing impressive smoker benefits that are shown in text (smoking status being the most reliable parameter). However, this finding is not even mentioned in the Abstract section of this particular publication.

The smoking cessation dogma does not help to initiate studies and to evaluate nicotine or other tobacco products in the role of a potential immunotherapy sensitizer. Transdermal nicotine has already been found to be highly effective in attenuating life-threatening cardiac dysfunction triggered by severe burns. ${ }^{8}$ Therapeutic benefits were associated with anti-inflammatory and immunomodulating effects of nicotine in this study. ${ }^{8}$ Nicotine has been demonstrated to interact with a target-the $\alpha 7$ nicotinic acetylcholine receptor ( $\alpha 7 \mathrm{nAChR})$-for attenuation of proinflammatory cytokine release. ${ }^{9}$ This group of receptors is found on $\mathrm{T}$ and $\mathrm{B}$ lymphocytes, macrophages, monocytes, and dendritic cells. ${ }^{10}$ Cessation of smoking might not be justified for immunotherapy patients since nicotine might be acting as a sensitizer as long as it is applied. It is a fact well known that activation of $\alpha 7 n A C h R s$ using nicotine elicits a transient increase of $\mathrm{Ca}^{2+}$ ions in immune cells which then can be rapidly desensitized.

The mainstream concept explaining advantageous monoimmunotherapy results in smokers suggest that stable genomic transformation is induced. ${ }^{6}$ However, genomic transformation is possibly not the only cause of tobacco product-induced cellular transformation. The overwhelming evidence shows that very short exposure of tumor cells to nicotine, carcinogens, or cytotoxic drugs can elicit a dramatic upregulation of PD-1/PD-L1. ${ }^{4}$ So, the mutation per se might not be the only basis of tobaccoinduced therapeutic aid and there might exist several epigenomic factors including 'metronomic smoking'. Should this transient upregulation be discontinued? We suggest that the fine-tuning of immunomodulation by compounds binding the nAChRs on immunocytes should be the focus of researchers.

The unwillingness of clinicians to suggest the continuation of smoking to patients is well motivated, since chronic obstructive lung disease (COPD) and NSCLC are both caused by smoking and often occur as a comorbidity. Both diseases involve rather complex aberrant immune functions which also include the PD-1/PD-L1 axis. Moderateto-severe COPD tended to have a more positive impact on the survival of patients with NSCLC as compared with the non-COPD group when treated with anti-PD-1 in a recent study. ${ }^{11}$ Therefore, the safety of continuation of smoking in preselected patients with COPD might not be jeopardized if a clinical trial including a no cessation group is initiated. The anti-PD-1 treatment can also decrease lung damage and neutrophilic inflammation as was demonstrated in recent experiments with mice that were chronically exposed to cigarette smoke. Since urological malignancies were also demonstrated to benefit from smoking in an anti-PD1 treatment paradigm, ${ }^{7}$ a careful selection of patients with mild COPD can be advocated for an investigational study. It is hard to predict the best candidate(s) for the role of immunosensitizers from the excess of 4000 toxins found in tobacco products. Lymphocytic nAChR stimulation with nicotine dissipates as quickly as its effect on brain cells due to a similar mechanism of action. The duration of smoking-related reward sensation (positive reinforcement) is a good timing estimate potentially applicable to the duration of leukocyte membrane 'cravings for nicotine'. Most leukocytes are short lived, with an average life span ranging from a day (neutrophils) to a few months (long-lived lymphocytes). Therefore, the accumulation of compounds mediating danger signals inside of leukocytes must not be significant 
within few months' time frame. A compartment of very long-lived T cells is minute. Cannot the idea of the continuation of immunocyte exposure to danger signals be a working hypothesis for further studies?

Funding The authors have not declared a specific grant for this research from any funding agency in the public, commercial or not-for-profit sectors.

Competing interests None declared.

Patient consent for publication Not required.

Provenance and peer review Not commissioned; externally peer reviewed.

Open access This is an open access article distributed in accordance with the Creative Commons Attribution Non Commercial (CC BY-NC 4.0) license, which permits others to distribute, remix, adapt, build upon this work non-commercially, and license their derivative works on different terms, provided the original work is properly cited, appropriate credit is given, any changes made indicated, and the use is non-commercial. See http://creativecommons.org/licenses/by-nc/4.0/.

\section{REFERENCES}

1 Gounant V, Duruisseaux M, Soussi G, et al. Does very poor performance status systematically preclude single agent anti-PD-1 immunotherapy? A multicenter study of 35 consecutive patients. Cancers 2021;13:1040-55.
2 Mo J, Hu X, Gu L, et al. Smokers or non-smokers: who benefits more from immune checkpoint inhibitors in treatment of malignancies? An up-to-date meta-analysis. World J Surg Oncol 2020;18:15-27.

3 D'Incecco A, Andreozzi M, Ludovini V, et al. PD-1 and PD-L1 expression in molecularly selected non-small-cell lung cancer patients. Br J Cancer 2015;112:95-102.

4 Wang G-Z, Zhang L, Zhao X-C, et al. The aryl hydrocarbon receptor mediates tobacco-induced PD-L1 expression and is associated with response to immunotherapy. Nat Commun 2019;10:1125-38.

5 Brouwers EEM, Huitema ADR, Beijnen JH, et al. Long-term platinum retention after treatment with cisplatin and oxaliplatin. BMC Clin Pharmacol 2008;8:1-10.

6 Bastida G, Beltrán B. Ulcerative colitis in smokers, non-smokers and ex-smokers. World J Gastroenterol 2011;17:2740-7.

7 Bellmunt J, de Wit R, Vaughn DJ, et al. Pembrolizumab as secondline therapy for advanced urothelial carcinoma. $N$ Engl $J$ Med 2017;376:1015-26.

8 Claassen L, Papst S, Reimers K, et al. Transdermal nicotine application attenuates cardiac dysfunction after severe thermal injury. Biomed Res Int 2015;2015:292076.

9 Hoover DB. Cholinergic modulation of the immune system presents new approaches for treating inflammation. Pharmacol Ther 2017;179:1-16.

10 Fujii T, Mashimo M, Moriwaki Y, et al. Physiological functions of the cholinergic system in immune cells. J Pharmacol Sci 2017;134:1-21.

11 Zhou J, Chao Y, Yao D, et al. Impact of chronic obstructive pulmonary disease on immune checkpoint inhibitor efficacy in advanced lung cancer and the potential prognostic factors. Trans/ Lung Cancer Res 2021;10:2148-62. 